\title{
DEPRESSÃO E PANDEMIA: ESTUDO COM UNIVERSITÁRIOS BRASILEIROS
}

\author{
Maria Eduarda Sandin Machado \\ Centro Universitário de Volta Redonda - UniFOA - Brasil \\ mariaeduardanutri.s@gmail.com \\ Bruna Casiraghi \\ Centro Universitário de Volta Redonda - UniFOA - Brasil \\ bruna.casiraghi@foa.org.br
}

Recepción Artículo: 10 mayo 2021 Admisión Evaluación: 10 mayo 2021 Informe Evaluador 1: 18 mayo 2021 Informe Evaluador 2: 25 mayo 2021 Aprobación Publicación: 01 junio 2021

\section{RESUMO}

A depressão é uma doença incapacitante, crônica e recorrente. Tem como sintomas humor deprimido, desprazer em atividades que antes geravam prazer, perda ou ganho significativo de peso por alteração no apetite, insônia ou hipersonia, falta de energia, sentimento de culpa, dificuldade de se concentrar, baixa autoestima, irritabilidade, ansiedade, e, em casos graves, pode levar ao suicídio. Pode ser classificadas como leve, moderada ou grave de acordo com a intensidade dos síntomas. Essa doença pode piorar em diversos contextos, como em situações de isolamento social, devido ao estresse, ansiedade, falta ou excesso de informação. Os universitários são afetados devido a mudança na rotina e a pressão psicológica. 0 objetivo do trabalho foi avaliar a quantidade de universitários com sintomas de depressão, comparando cursos da área da saúde com 0 de outras áreas. A pesquisa teve uma amostra de 210 universitários do estado do Rio de Janeiro, Brasil, sendo $69 \%$ do gênero feminino e 31\% masculino, com idade média de 23 anos. 0 questionário foi enviado via rede social contendo: um formulário avaliando dados pessoais e o Inventário de Depressão de Beck (BDI). 0 BDI mostrou em relação aos universitários que 119 (56.7\%) não apresentaram sintomas, 20 (9.5\%) apresentaram disforia e 61 (29\%) apresentaram sintomas de depressão. Conclui-se que os dados indicaram altas taxas de sintomas depressivos entre os universitários estudados e a necessidade de ações de acompanhamento.

Palavras chave: depressão; estudante universitário; pandemia

\section{ABSTRACT}

Depression and pandemia: study with brazilian university students. Depression is a disabling, chronic and recurrent disease. Symptoms include depressed mood, displeasure in activities that previously generated pleasure, loss or significant weight gain due to changes in appetite, insomnia or hypersomnia, lack of ener- 


\section{DEPRESSÃO E PANDEMIA: ESTUDO COM UNIVERSITÁRIOS BRASILEIROS}

gy, guilt, difficulty concentrating, low self-esteem, irritability, anxiety, and, in severe cases, can lead to suicide. It can be classified as mild, moderate or severe according to the intensity of the symptoms. This disease can worsen in several contexts, such as in situations of social isolation, due to stress, anxiety, lack or excess information. College students are affected due to the change in routine and psychological pressure. The objective of the work was to evaluate the number of university students with symptoms of depression, comparing courses in the health field with those in other areas. The research had a sample of 210 university students from the state of Rio de Janeiro, Brazil, 69\% female and 31\% male, with an average age of 23 years. The questionnaire was sent via social network containing: a form evaluating personal data and the Beck Depression Inventory (BDI). In relation to university students, the BDI showed that $119(56.7 \%)$ had no symptoms, 20 (9.5\%) had dysphoria and 61 (29\%) had symptoms of depression. It is concluded that the data indicated high rates of depressive symptoms among the university students studied and the need for follow-up actions.

Keywords: depression; university students; pandemia

\section{INTRODUÇÃO}

Antes chamada melancolia por Hipócrates, a depressão teve sua descoberta no séc. XIX, quando Wilhelm Griesinger declara que "doenças mentais são doenças do cérebro" (Gonçales \& Machado, 2007). Essa é hoje considerada uma doença crônica e recorrente, mais frequente em mulheres, de etiologia multifatorial podendo ser causada por genética, bioquímica cerebral e/ou eventos vitais. Estima-se que no Brasil 10 milhões de pessoas sofrem de depressão (Fleck et al., 2009; Brasil, 2019; OMS, 2018; Sezini \& Gil, 2014).

Principal causa de incapacidade do mundo, a depressão é considerada uma doença que representa grande risco para a saúde pública. Seus sintomas são: humor deprimido, desprazer em atividades que antes geravam prazer, perda ou ganho significativo de peso por alteração no apetite, insônia ou hipersonia, falta de energia, sentimento de culpa, dificuldade de se concentrar, baixa autoestima, irritabilidade, ansiedade, e, em casos graves, pode levar ao suicídio. 0 diagnóstico da depressão deve considerar a apresentação de cinco ou mais sintomas por pelo menos 2 semanas e pode ser classificada, de acordo com a intensidade dos sintomas, em leve, moderada ou grave (APA, 2014; OMS, 2018).

Devido à falta de treinamento de médicos não psiquiatra, a depressão ainda é subdiagnosticada e, quando diagnosticada corretamente, nem sempre a proposta de tratamento é correta (Fleck et al., 2009; Dartora; Pozzebon \& Bitello, 2013). Por ser uma condição multifatorial, que envolve diferentes aspectos e conjunturas, o tratamento precisa combater os diversos fatores de aparecimentos e envolvidos. Além disso, a adesão ao tratamento na depressão pode ser dificultada devido aos efeitos colaterais das medicações utilizadas.

A depressão pode, também, ser agravada em diversos contextos, como por exemplo na pandemia. Um estudo realizado na China mostrou que pessoas com alto nível socioeconômico tem mais informações referente ao COVID-19 e, consequentemente, adotam mais medidas de proteção. Porém, pessoas vulneráveis, com baixo acesso a informações, podem ter práticas preventivas inadequadas e tendem a desenvolver quadros depressivo (Zhong et al., 2020). Outro estudo mostrou que pessoas com acesso a informações fidedignas, baseadas em evidências, apresentam menores chances de sofrer impacto psicológico (Wang et al., 2020).

Barros e colaboradores (2020) realizaram um estudo com brasileiros durante a pandemia avaliando os relatos de humor e identificaram que $40 \%$ dos brasileiros apresentaram tristeza e depressão, mais de $50 \%$ apresentaram nervosismo e ansiedade e 40\% relataram problema de sono, sendo esses sintomas mais significativos em adultos jovens (entre 18 e 29 anos). Estudo realizado na China, avaliando 0 impacto psicológico da pandemia, mostrou que $16,5 \%$ dos entrevistados sofreram com sintomas depressivos graves e moderados, $28,8 \%$ relataram ter sentido sintomas de ansiedade e 8,1\% relataram estresse (Wang et al., 2020).

Os estudos sobre o percurso de universitários indicam que existem especificidades neste processo que levam a preocupações com a saúde mental destes indivíduos (Aragão et al., 2017; Costa et al., 2012). Os universitários vivenciam experiências inerentes a vida acadêmica, que envolve as exigências da sociedade, de ser eficaz, adaptar-se às pressões, ser "alguém na vida", e ainda a adaptação às mudança do cotidiano, as provas e 
trabalhos, a carga de estudos e a novas formas de estudar (Almeida, 2007; Dinis, 2013). Frente a essas questões, ressalta-se a necessidade de acompanhamento dos estudantes do ensino superior quanto às questões relativas à saúde mental, visto que já são uma população de risco e pode resultar no abandono dos estudos (Lelis et al, 2020).

\section{OBJETIVOS DA INVESTIGAÇÃO}

0 objetivo do presente trabalho foi avaliar a prevalência de depressão em universitários no período de isolamento social e, ainda, comparar o aparecimento de sintomas depressivos em universitários de cursos da saúde e de outros cursos.

\section{AMOSTRA E PARTICIPANTES}

Participaram da pesquisa 210 universitários do estado do Rio de Janeiro, sendo 145 (69.0\%) do sexo feminino e 65 (31.0\%) do sexo masculino, idade média de 23.17 anos ( $\mathrm{DP}=4.13$, mínima de 18 e máxima de 40 anos). Dos participantes, 127 (60.5\%) graduandos eram de cursos da área da saúde (Nutrição, Psicologia, Enfermagem, Educação Física, Medicina, Medicina Veterinária e Odontologia); e 83 (39.5\%) graduandos de cursos de outras áreas (Direito, Engenharia Mecânica, Engenharia Civil, Engenharia Metalúrgica, Engenharia Elétrica, Engenharia de Agronegócio, Administração, Pedagogia, Serviço Social, Jornalismo, Arquitetura e Urbanismo, Publicidade e Propaganda, Design, Gestão e Empreendedorismo, Gestão de Recursos Humanos e Sistema de Informação). Foram excluídos alunos com idade menor que 18 anos, deficiência sensorial ou de comunicação e que não cursavam ensino superior.

\section{METODOLOGIA E INSTRUMENTOS UTILIZADOS}

A pesquisa foi realizada com universitários através de formulário disponibilizado eletronicamente e sem a possibilidade de identificação dos participantes. 0 projeto de pesquisa foi aprovado pelo Comitê de Ética em Pesquisa sob registro CAAE $n^{0}$ 31220620.0.0000.5237 e antes de iniciar a resposta o participante declarava concordar com o Termo de consentimento livre e esclarecido (TCLE).

A coleta de dados ocorreu entre junho e julho de 2020, durante o período de pandemia do SARS-CoV-2 (COVID-19), que se caracteriza por uma doença infecciosa, que teve sua descoberta na China no ano de 2019, e em 11 de março de 2020 foi determinada como uma pandemia pela OMS (OMS, 2020). 0 Brasil passou recomendações através do governo federal pela portaria no 340, de 30 de março de 2020, dentre elas, 0 isolamento social, no qual recomenda-se que indivíduos sintomáticos ou com suspeita do vírus deveriam ser isolados para impedir a disseminação (Pereira et al, 2020). Frente às dificuldades de contato social, a coleta se deu através de divulgação no Whatsapp, Facebook, Twitter e Instagram.

A pesquisa era composta por 2 partes: dados pessoais e Inventário de depressão de Beck (BDI). Os dados pessoais solicitados tiveram como objetivo coletar dados básicos demográficos como: idade, sexo, curso que frequenta e período que cursa na universidade. Visto que o período de pandemia favorece o surgimento de sintomas isolados, ansiedade, depressão e Transtorno de Estresse pós traumático (PTSD) (Ramírez-Ortiz, et al, 2020), foi perguntado aos participantes se eles sentem que durante 0 isolamento social o humor foi alterado.

0 Inventário de Depressão de Beck (BDI) (Gorestein, 1998) é um teste de rastreamento de sintomas depressivos, e não de diagnóstico. Consiste em 21 itens, incluindo sintomas e atitudes, cuja intensidade varia de 0 a 3. Os itens da escala referem-se a tristeza, pessimismo, sensação de fracasso, falta de satisfação, sensação de culpa, sensação de punição, autodepreciação, autoacusações, ideias suicidas, crises de choro, irritabilidade, retração social, indecisão, distorção da imagem corporal, inibição para o trabalho, distúrbio do sono, fadiga, perda de apetite, perda de peso, preocupação somática, diminuição de libido. Para a categorização dos resultados, foi utilizada a classificação para pessoas não diagnosticadas, de Kendall (1987), que estabelece os seguintes parâmetros para análise dos escores: de 0 a 15 é considerado sem sintomas, de 15 a 20 classifica-se como disforia e acima de 20 apresenta sintomas depressivos. 


\section{DEPRESSÃO E PANDEMIA: ESTUDO COM UNIVERSITÁRIOS BRASILEIROS}

Os dados foram analisados estatisticamente tomando os estudantes agrupados pelas suas variáveis pessoais. Para a análise de dados, utilizou-se o programa IBM SPSS 26. Realizou-se análises de estatística descritiva e comparação de médias (teste t-Student, coeficiente de correlação de Sperman, ANOVA e teste de Tukey). 0 nível de significância adotado foi de $5 \%$, ou seja, p-valor $£ 0.05$.

\section{RESULTADOS ALCANÇADOS}

Os resultados indicam índices preocupantes com relação tanto a percepção das mudanças de humor como dos escores do inventário utilizado. Quando perguntados sobre o humor durante a pandemia, 148 (70.5\%) dos universitários afirmaram sentir mudança e 62 (29.5\%) negaram mudança de humor durante a pandemia. A análise dos sintomas depressivos avaliados através do BDI indicou que 119 (56.7\%) não apresentaram sintomas, 20 (9.5\%) apresentaram disforia e 61 (29\%) apresentaram sintomas de depressão.

A comparação dos sintomas depressivos entre os sexos indicou uma diferença significativa e maior entre as mulheres, com média de 17,54 ( $t=-3.13, p=0.02$ ) pontos, média essa que se configura como disforia (entre 1520); já entre os homens a média foi de 12,08 pontos, classificação que representa sem sintomas depressivos (entre 0 e 15 pontos).

Ao avaliar os escores do BDI com grupos etários (até 20 anos; entre 21 e 24 anos e maior que 25 anos), observou-se que os mais jovens possuem índices mais altos, apresentando uma média de 22.95 que se enquadra entre o nível de risco para sintomas depressivos, e significativamente maior que as outras faixas etárias ( $Z=9.77$, p<0.001), cujos índices diminuíam conforme a idade aumentava, obtendo médias iguais a 14.81 e 12.22, respectivamente (Figura 1).

Figura 1- Gráfico de médias do escore do BID por faixas etárias

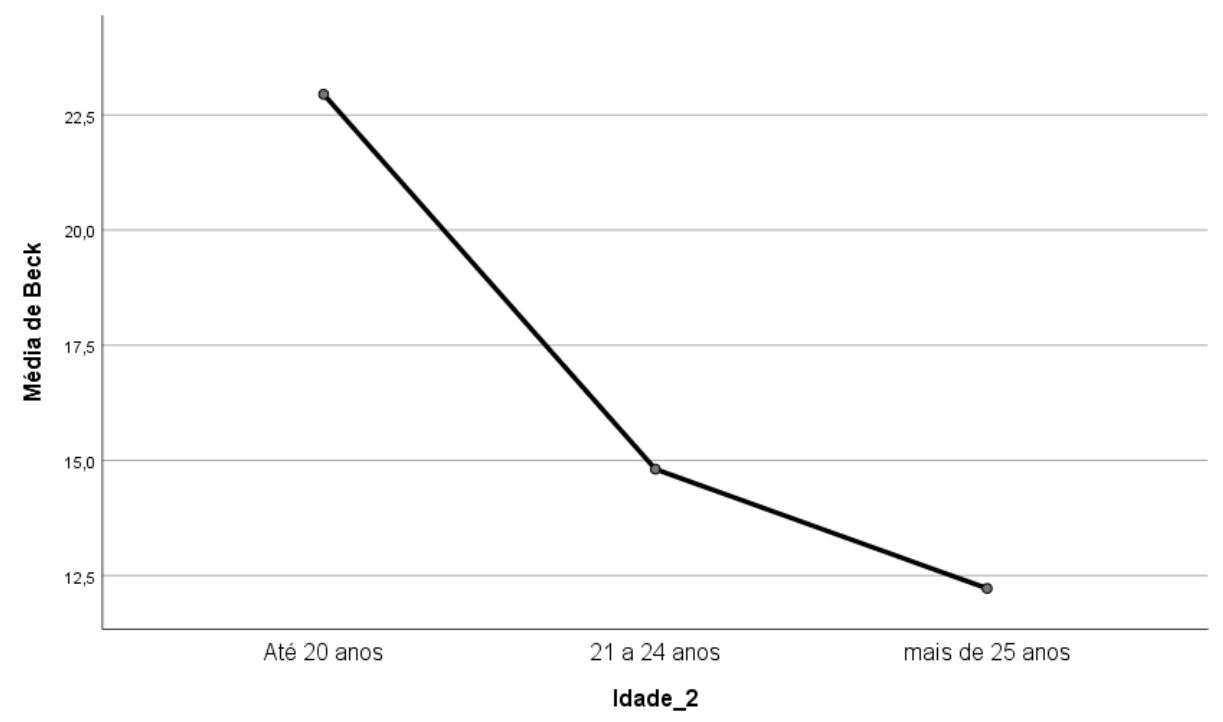

A comparação entre estudantes da área da saúde e de outras áreas não apontou diferença significativa entre os grupos. 


\section{DISCUSSÃO}

Os dados encontrados indicam taxas de depressão maiores que os outros estudos realizados durante a pandemia com a população em geral (Barros et al., 2020; Wang et al., 2020), o que pode ser explicado pelo fato de serem universitários e a pandemia significar uma preocupação maior frente as expectativas de futuro, como, por exemplo, conclusão do curso e inserção no mercado de trabalho. 0 fato de mais de $70 \%$ dos entrevistados relatarem perceber mudanças de humor durante a pandemia indicam para a necessidade de acompanhamento, buscando compreender de que forma e em que intensidade tais mudanças aconteceram, assim como ações de apoio e suporte.

A comparação dos resultados obtidos com outros estudos realizados pré-pandemia indica que os estudantes avaliados no ano de 2020 apresentam índices de sintomas depressivos maiores. Estudos anteriores indicavam sintomas depressivos entre 5\% e 7\% dos universitários (Maltoni et al., 2019; Padovani e et al., 2014), enquanto os dados obtidos durante a pandemia apontam para uma taxa de 29\% de universitários com sintomas depressivos, índice cinco vezes maior. É importante destacar que a comparação com os dados anteriores fica prejudicada, pois trata-se de um formulário de autorrelato e as condições ambientais e sociais diferem. No entanto, os resultados encontrados, por si só, já indicam uma realidade preocupante e que necessita de atenção e cuidado. Para além dos fatores já conhecidos como exigência acadêmica, medo de fracassar, somam-se, no contexto atual da pandemia, a incerteza quanto ao futuro e a preocupação com a saúde, o que pode explicar o número aumentado de sintomas depressivos nesta população.

Tais dados estão relacionados, também, com 0 fato de os estudantes mais jovens apresentarem resultados mais preocupantes com relação à sintomas depressivos, o que pode estar relacionado ao processo de adaptação ao ensino superior, às exigências acadêmicas, à alteração na rotina, ao início da vida adulta, ao medo de fracassar ou à cobrança de "ser alguém" na vida (Krefer et al., 2019).

As mulheres estudadas apresentaram resultados mais altos, indicando maior propensão que os homens a apresentar sintomas depressivos. Os dados corroboram estudos da área que têm mostrado prevalência de depressão em mulheres, e como fatores explicativos tem sido apresentado o fato de a mulher procurar mais atendimento de saúde, mudanças hormonais e fisiológicas e a entrada da mulher no mercado de trabalho (Rombaldi et al., 2010). Um estudo realizado em 10 países mostrou que, no Brasil, a chance de as mulheres desenvolverem a depressão é de 2.6 vezes maior quando comparadas aos homens (Bromet et al., 2011).

A comparação de sintomas depressivos entre estudantes da saúde e das outras áreas não se mostrou significativo neste estudo, embora em estudos anteriores tais diferenças tenham sido observadas (Barroso, Oliveira \& Andrade, 2019; Nascimento, 2020). Frente aos altos índices de sintomas depressivos detectados e ao contexto de pandemia, a área de estudo dos acadêmicos parece não ser uma variável relevante.

\section{CONCLUSÕES}

0 presente trabalho buscou analisar a prevalência de depressão entre os universitários no período de isolamento social. Considerando que o estudo foi realizado no contexto da pandemia do Covid-19, esbarra-se com as impossibilidades de comparação dos dados e extrapolações para outros contextos e cenários. Frente a uma situação extraordinária e não vivida anteriormente pelos indivíduos pesquisados, dados referentes aos sintomas depressivos e comportamento alimentar dessa população são únicos e devem ser considerados dentro do contexto.

Os dados obtidos indicam altas taxas de sintomas depressivos entre os universitários estudados, além de relatarem percepção de mudança de humor durante a pandemia, o que aponta para a necessidade de ações nas instituições de ensino superior e, também, de políticas públicas que enfoquem esta população.

Destaca-se a importância do acompanhamento da saúde mental desta parcela da população, enfatizando a necessidade de realização de outros estudos sobre depressão e saúde mental em universitários, dentro e fora do contexto de pandemia, permitindo conhecer melhor a realidade e propor estratégias de prevenção, acompanhamento e tratamento. 


\section{DEPRESSÃO E PANDEMIA: ESTUDO COM UNIVERSITÁRIOS BRASILEIROS}

\section{REFERENCIAS BIBLIOGRÁFICAS}

Almeida, L. S. (2007). Transição, adaptação académica e êxito escolar no ensino superior. Revista galego-portuguesa de psicoloxía e educación,15,13.

American Psychiatric Association. (2014). DSM-5: Manual diagnóstico e estatístico de transtornos mentais. 5.ed. Artmed Editora.

Aragão, J. C. D. P., Casiraghi, B., Mota, É. M., Abrahão, M. A. B., De Almeida, T. A., Baylão, A. C. D. P., \& Araújo, P. A. M. T. (2017). Saúde mental em estudantes de medicina. Revista de Estudios e Investigación en Psicología y Educacion, 14, 038.

Barroso, S. M., Oliveira, N. R. D., \& Andrade, V. S. D. (2019). Solidão e Depressão: Relações com Características Pessoais e Hábitos de Vida em Universitários. Psicologia: Teoria e pesquisa, 35

Barros, M. B. D. A., Lima, M. G., Malta, D. C., Szwarcwald, C. L., Azevedo, R. C. S. D., Romero, D., ... \& Gracie, R. (2020). Relato de tristeza/depressão, nervosismo/ansiedade e problemas de sono na população adulta brasileira durante a pandemia de COVID-19. Epidemiologia e Serviços de Saúde, 29, e2020427.

Brasil. Ministério da Saúde. (2020). Depressão: como diagnosticar, quais os sintomas e qual o tratamento. 2019. Disponível em: <https://saude.gov.br/saude-de-a-z/depressa0>. Acesso em: 19 mar. 2020

Bromet, E., Andrade, L. H., Hwang, I., Sampson, N. A., Alonso, J., De Girolamo, G., ... \& Kessler, R. C. (2011). Cross-national epidemiology of DSM-IV major depressive episode. BMC medicine, 9(1), 1-16.

Costa, E. F. de 0., Santana, Y. S., de Abreu Santos, A. T. R., Martins, L. A. N., de Melo, E. V., \& de Andrade, T. M. (2012). Sintomas depressivos entre internos de medicina em uma universidade pública brasileira. Revista da Associação Médica Brasileira, 58(1), 53-59.

Dartora, B. Z., Pozzebon, Â., \& Bitello, A. (2013). Relação entre depressão, alimentação e o número de internações hospitalares no hospital Santa Isabel de progresso-RS. Revista Destaques Acadêmicos, 5(3).

de Cássia Lelis, K., Brito, R. V., de Pinho, S., \& Pinho, L. (2020). Sintomas de depressão, ansiedade e uso de medicamentos em universitários. Portuguese Journal of Mental Health Nursing/Revista Portuguesa de Enfermagem de Saude Mental, (23).

Dinis, A. C. A. R. (2013). Adaptação académica, apoio social e bem-estar subjetivo dos estudantes do ensino superior: Um estudo nas residências universitarias [Universidade de Coimbra].

do Nascimento, J. R. P., Graça, Á., de Souza Minante, D. W., Bertolin, J. N. G., Fonseca, H. S., de Oliveira, D. V., ... \& do Nascimento, M. A. (2020). Fatores associados à ansiedade e depressão em estudantes universitários. Research, Society and Development, g(9), e562997533-e562997533.

Fleck, M. P., Berlim, M. T., Lafer, B., Sougey, E. B., Porto, J. A. D., Brasil, M. A., .. \& Hetem, L. A. (2009). Revisão das diretrizes da Associação Médica Brasileira para o tratamento da depressão. Brazilian journal of psychiatry, 31, S7-S17.

Gonçales, C. A. V., \& Machado, A. L. (2007). Depressão, o mal do século: de que século?. Rev. enferm. UERJ, 298-304.

Gorenstein, C., \& Andrade, L. H. S. G. (1998). Inventário de depressão de Beck: propriedades psicométricas da versão em português. Rev psiq clin, 25(5), 245-50.

Krefer, L., \& vayego, S. A. (2019). Prevalência de sintomas depressivos em estudantes universitários. Cadernos Brasileiros de Saúde Mental/Brazilian Journal of Mental Health, 11 (28), 170-181.

Maltoni, J., de Camargo Palma, P., \& Neufeld, C. B. (2019). Sintomas ansiosos e depressivos em universitários brasileiros. Psico, 50(1), e29213-e29213.

Organização Mundial da Saúde - OMS. (2018) Depressão. Disponível em: $<$ <ttps://www.paho.org/bra/index.php?option=com_content\&view=article\&id=5635:fol ha-informativadepressa0\&ltemid=1095>. Acesso em: 19 mar. 2020.

Padovani, R. D. C., Neufeld, C. B., Maltoni, J., Barbosa, L. N. F., Souza, W. F. D., Cavalcanti, H. A. F., \& Lameu, J. D. N. (2014). Vulnerabilidade e bem-estar psicológicos do estudante universitário. Revista brasileira de terapias cognitivas, 10(1), 02-10. 
Pereira, M. D., de Oliveira, L. C., Costa, C. F. T., de Oliveira Bezerra, C. M., Pereira, M. D., dos Santos, C. K. A., \& Dantas, E. H. M. (2020). A pandemia de COVID-19, o isolamento social, consequências na saúde mental e estratégias de enfrentamento: uma revisão integrativa. Research, Society and Development, 9(7), e652974548-e652974548.

Ramírez-Ortiz, J., Castro-Quintero, D., Lerma-Córdoba, C., Yela-Ceballos, F., \& Escobar-Córdoba, F. (2020). Consecuencias de la pandemia Covid 19 en la salud mental asociadas al aislamiento social.

Rombaldi, A. J., Silva, M. C. D., Gazalle, F. K., Azevedo, M. R., \& Hallal, P. C. (2010). Prevalência e fatores associados a sintomas depressivos em adultos do sul do Brasil: estudo transversal de base populacional. Revista Brasileira de Epidemiologia, 13(4), 620-629.

Sezini, A. M., \& do Coutto Gil, C. S. G. (2014). Nutrientes e depressão. Vita et Sanitas, 8(1), 39-57.

Wang, C., Pan, R., Wan, X., Tan, Y., Xu, L., Ho, C. S., \& Ho, R. C. (2020). Immediate psychological responses and associated factors during the initial stage of the 2019 coronavirus disease (COVID-19) epidemic among the general population in China. International journal of environmental research and public health, 17(5), 1729.

Zhong, B. L., Luo, W., Li, H. M., Zhang, Q. Q., Liu, X. G., Li, W. T., \& Li, Y. (2020). Knowledge, attitudes, and practices towards COVID-19 among Chinese residents during the rapid rise period of the COVID-19 outbreak: a quick online cross-sectional survey. International journal of biological sciences, 16(10), 1745. 
